\title{
Turbulence in circumstellar disks
}

\author{
F. Hersant ${ }^{1,2,3}$, B. Dubrulle ${ }^{1}$, and J.-M. Huré ${ }^{4,5}$ \\ ${ }^{1}$ CNRS URA 2464 GIT/SPEC/DRECAM/DSM, CEA Saclay, 91191 Gif-sur-Yvette Cedex, France \\ e-mail: bdubrulle@cea.fr \\ 2 LESIA CNRS UMR 8109, Observatoire de Paris-Meudon, Place Jules Janssen, 92195 Meudon Cedex, France \\ 3 Institut für Theoretische Astrophysik, Tiergartenstraße 15, 69121 Heidelberg, Germany \\ ${ }^{4}$ LUTh CNRS UMR 8102, Observatoire de Paris-Meudon, Place Jules Janssen, 92195 Meudon Cedex, France \\ 5 Université Paris 7 Denis Diderot, 2 Place Jussieu, 75251 Paris Cedex 05, France
}

Received 31 January 2003 / Accepted 16 August 2004

\begin{abstract}
We investigate the relationship between circumstellar disks and the Taylor-Couette flow. Using the Reynolds similarity principle, this results in a number of parameter-free predictions about stability of the disks, and their turbulent transport properties, provided the disk structure is available. We discuss how the latter can be deduced from interferometric observations of circumstellar material. We use the resulting disk structure to compute the molecular transport coefficients, including the effect of ionization by the central object. The resulting control parameter indicates that the disk is well into the turbulent regime. The analogy is also used to compute the effective accretion rate, as a function of the disk characteristic parameters (orbiting velocity, temperature and density). These values are in very good agreement with experimental, parameter-free predictions derived from the supposed relationship. The turbulent viscosity is also computed and found to correspond to an $\alpha$-parameter $2 \times 10^{-4}<\alpha<2 \times 10^{-2}$. Predictions regarding fluctuations are also checked: luminosity fluctuations in disks do obey the same universal distribution as energy fluctuations observed in a laboratory turbulent flow. Radial velocity dispersion in the outer part of the disk is predicted to be of the order of $0.1 \mathrm{~km} \mathrm{~s}^{-1}$, in agreement with available observations. All these issues provide a proof of the turbulent character of circumstellar disks, as well as a parameter-free theoretical estimate of effective accretion rates.
\end{abstract}

Key words. turbulence - Solar system: formation - stars: formation - accretion, accretion disks

\section{Introduction}

Stars form by gravitational collapse of molecular clouds. During this process, proto-stars get surrounded and plausibly are fed by an envelope/disk made of gas and dust, which can, under certain conditions, coagulate to form planetary embryos. There is little doubt that gas motion, usually considered as turbulent, plays a major role. Turbulent motion enhances transport properties, thereby accelerating the evolution of the temperature and density in the envelope/disk. Also, turbulence may catalyze planet formation thanks to the trapping of dust particle inside large-scale vortices (Barge \& Sommeria 1995; Tanga et al. 1996; Chavanis 2000). As of now, the assertion that circumstellar disks are turbulent (what we shall refer to as the "turbulent hypothesis") has never been properly checked. It mainly relies on the fact that the luminosity produced by the disk interacting with the central star is very large (see e.g. Hartmann et al. 1998). In certain cases (FU Orionis-type systems), this luminosity is so high that it even supersedes the stellar component. The most widely accepted scenario so far to account for the abnormal luminosities of young stellar objects involves a magnetospheric accretion for classical T Tauri stars. In this case, the matter in the inner parts of the disk is coupled with the stellar magnetic field and falls onto the stars along the field lines at the free fall velocity (see e.g. Gullbring et al. 1998; Muzerolle et al. 1998). This entails an accretion shock at the stellar surface in which almost all the visible and UV excess is produced (veiling the stellar lines). This scenario has shown many successes in interpreting spectral features of $\mathrm{T}$ Tauri stars like sodium and hydrogen line profiles (see e.g. Muzerolle et al. 1998). In the case of Fu Ori stars, the currently advocated picture involves a wide boundary layer (see e.g. Popham et al. 1996). One of the main weaknesses of this scenario is its low predictive power since it relies on an adjustable parameter (the accretion rate) which must be postulated a posteriori by comparison with observational data.

Indeed, in this framework, the luminosity is directly related to the amount of energy released by the disk. The necessity for turbulence comes from the hypothesis that no laminar motions can produce the amount of energy dissipated required to explain observed luminosities (see e.g. Pringle 1981 and references therein). However, no attempt has been made to substantiate this claim in a quantitative manner. The questions we address here are: what are the luminosities produced by a laminar disk and by a turbulent disk? How do these compare with observations? These two questions are equally difficult to answer, but they hide different levels of difficulties. One is of a theoretical nature: the physical processes at work in 
this disk/star interaction region are complex. A correct description should simultaneously include the resolution of turbulence (with compressibility effects), radiative transfer (accounting for UV-irradiation by the star), magnetic processes, chemistry, the disk flaring, phase separation, time evolution, etc. The second difficulty is of an observational nature. At the present time, information has been gathered about the temperature, density and velocity distributions in the outer parts of disks, at $\gtrsim 100 \mathrm{AU}$ typically, thanks to high resolution interferometry and clever data analysis (Guilloteau \& Dutrey 1998). Unfortunately, basic parameters (mean free path, sound velocity and viscosity) connected with the gas dynamics and dissipation are still not known in the inner regions, and especially in the region where the disk and the star interact.

Because of these difficulties, we choose to adopt a radically different approach than the classical model: instead of trying to build a fully "realistic" circumstellar disk, we use a simplified hydrodynamic model ("zero order model") and study in detail its physical properties. In the future, we will slowly increase its complexity (and reality!) by adding new ingredients like magnetic field, stratification, radiative transfer. Here, we show that our zero order model is analogous to an incompressible rotating shear flow. It is therefore amenable to treatment as a simple laboratory prototype, the Taylor-Couette flow. From theoretical and experimental studies of the properties of this prototype, one can then build general laws in circumstellar disks by a simple use of the Reynolds similarity principle. Taylor-Couette flow is a classical laboratory flow, and it has been the subject of many experiments. A recent review about stability properties and transport properties for use in astrophysical flows has been made by Dubrulle et al. (2004). As a result, they derive critical conditions for stability, and simple scaling laws for transport properties, including the influence of stratification, magnetic field, boundary conditions and aspect ratio. In the present paper, we apply these results to circumstellar disks and derive the expression of the characteristic parameters of the model as a function of astrophysical observables. We propose a procedure of quantitative estimation of the observables using the observational results of Guilloteau \& Dutrey (1998) and derive parameter-free predictions about turbulence and turbulent transport in circumstellar disks. These predictions are tested against observational data from $\mathrm{T}$ Tauri and FU Ori stars.

\section{Hydrodynamic model}

\subsection{Basic ideas}

Observation of circumstellar disks suggests that they have sizes between 100 and 1000 astronomical units. In the following, we will focus only on the part of the disk expected to behave like an incompressible fluid. An estimate of the importance of compressibility can be obtained via the Mach number, the ratio of the typical velocity to thermal velocities. It is generally admitted that compressibility effects start playing a role when this number reaches values of unity. In the outer part of the disk, this ratio has been estimated by Guilloteau \& Dutrey (1998) from CO line profiles. Its value is about $0.2-0.3$. In the inner part of the disk (radius ranging from 1 to 30 astronomical units), we may use the disk structure inferred from the $\mathrm{D} / \mathrm{H}$ ratio measured in the Solar System (Drouart et al. 1999; Hersant et al. 2001), which leads to a Mach number of the order of 0.05 to 0.1 . These figures indicate that both the inner and the outer part can be treated as incompressible fluids. Closer to the star, the situation is less clear. On the one hand, temperature tends to increase strongly, leading to an increase of the sound velocity and a decrease of the Mach number. On the other hand, as one gets closer to the boundary, one may expect larger typical velocities induced by larger velocity gradients, and thus an increase of the Mach number. There are no direct observations supporting one scenario or the other. We shall then consider two scenarios: one in which the Mach number Ma never exceeds unity. In this case, the whole disk is incompressible, and connects smoothly onto the star at the star radius. The inner boundary is thus defined as $r_{\mathrm{i}}=r_{*}$. In the second scenario, the Mach number reaches unity at some "interaction radius" $r_{\text {in }}$, leading to an inner boundary at $r_{\mathrm{i}}=r_{\text {in }}$. At this location, a shock appears, in which all velocities are suddenly decreased to very small values. In the shock, all the kinetic energy is transfered to the thermal energy, thereby producing a strong temperature increase (by a factor of $1+M a^{2} \simeq 2$ ). This entails an increase of ionization and the matter gets more coupled to the stellar magnetic field. This second situation is considered in magnetospheric accretion models (Hartmann et al. 1998; Hartmann et al. 2002), in which case $r_{\text {in }}$ is the Alfven radius; see Schatzman $(1962,1989)$. Figure 1 summarizes these two possible configurations. From a hydrodynamical point of view, in the first situation the boundary is similar to a freeslip boundary (with possible non-zero velocities in the direction tangential to the star boundary), while in the second situation, the interaction radius acts as a no-slip boundary (with all velocities becoming zero). This difference may be reflected in the transport properties, see Dubrulle et al. (2004). In the following, the free-slip boundary condition will be referred to as smooth, while the no-slip boundary will be referred to as rough. In the laboratory experiments reviewed in Dubrulle et al. (2004), the turbulent transport depends on the boundary conditions. Specifically, transport is enhanced (with respect to other boundary conditions) for boundary conditions of the rough or no-slip type. In the astrophysical case, it is not quite clear whether these two boundary conditions apply, or even whether different inner and outer boundary conditions result in an intermediate transport enhancement. We shall therefore devise observational tests using quantities independent of boundary conditions via a suitable non-dimensionalisation.

\subsection{Basic equations}

In either case, the angular velocity $\Omega$ at the inner boundary is that of the star, namely $\Omega\left(r_{\mathrm{i}}\right)=\Omega_{*}$. For $r>r_{\mathrm{i}}$, the Mach number of the flow is less than one by construction, i.e. pressure fluctuations vary over a time scale short compared to the dynamical time. In such a case, one can assume hydrostatic equilibrium in the vertical direction, implying a decoupling of the vertical and horizontal structure. It is then convenient to describe the disk by its "horizontal equations", obtained by 


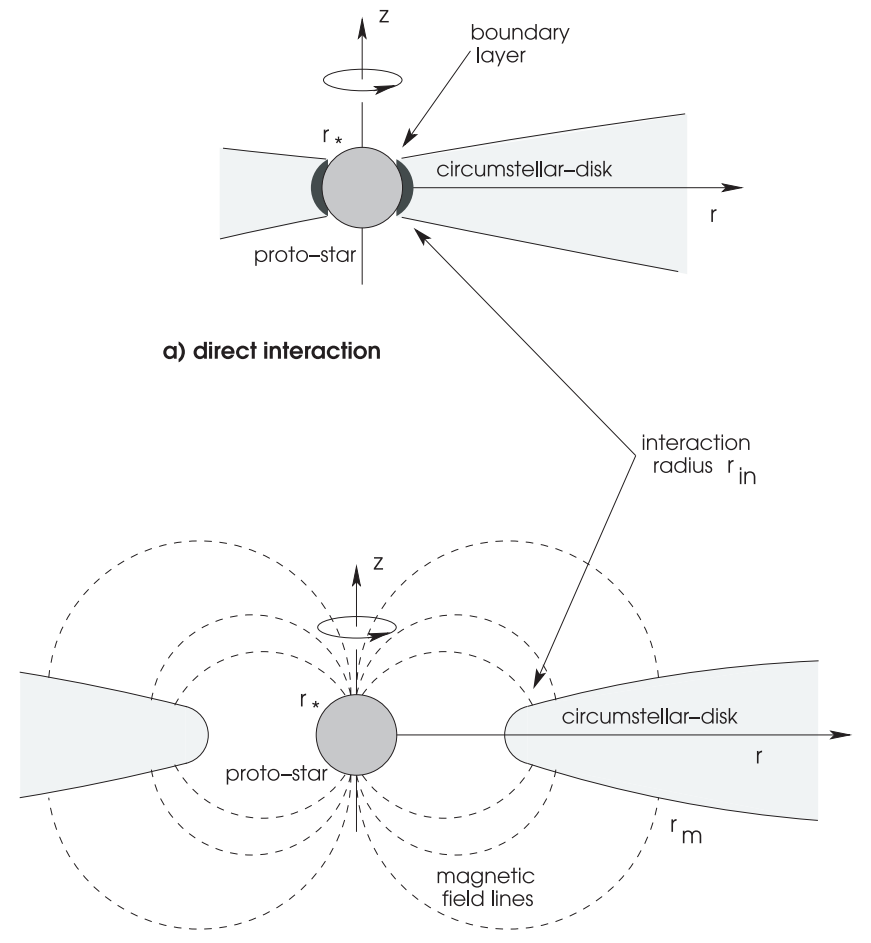

b) indirect interaction

Fig. 1. Two possible configurations considered in the present model: a) the whole disk is incompressible and extends onto the proto-star; and b) the disk is incompressible until an "interaction radius" imposed for instance by a magnetic field.

averaging the original equations of motion in the vertical direction. The procedure is described e.g. in Dubrulle (1992). It leads to:

$\partial_{t} \Sigma+\partial_{i}^{h} \Sigma u_{i}=0$

$\partial_{t}\left(\Sigma u_{i}\right)+\partial_{j}^{h}\left(\Sigma u_{i} u_{j}\right)=-\partial_{i}^{h} H p+\partial_{j}^{h} \tau_{i j}-\Sigma \frac{G M}{r^{2}} e_{r}$,

$\tau_{i j}=\mu\left(\partial_{i}^{h} u_{j}+\partial_{j}^{h} u_{i}\right)+\left(\zeta+\frac{\mu}{3}\right) \partial_{j}^{h} u_{j}$.

Here, $\partial^{h}$ is the horizontal gradient $\left(\partial_{z}=0\right), u$ and $p$ are the Favre average of the velocity and the pressure over the vertical direction, $\Sigma$ is the surface density, $\mu$ and $\zeta$ are surface viscosity coefficients, $G$ is the gravitational constant, $M$ the mass of the star, and $r$ the distance to the star in cylindrical coordinate, $e_{r}$ is a unit vector in the radial direction and $H$ the vertical scale height. In the hydrostatic approximation,

$H=c_{s}\left(\frac{r^{3}}{G M}\right)^{1 / 2}$,

where $c_{s}$ is the sound velocity. This expression is only valid when self-gravity can be neglected, namely when:

$\frac{M_{\text {disk }}}{M} \lesssim \frac{H}{R}$

where $M_{\text {disk }}$ and $M$ are the masses of the disk and the star, respectively (see e.g. Huré 2000).

In the opposite case, $H$ will rather vary like the Jeans length in the vertical direction, as:

$H=\frac{c_{s}^{2}}{4 \pi G \Sigma}$
These equations should be supplemented with an equation for the surface energy $E \sim H c_{s}^{2}$, but we shall not need it in the sequel.

\subsection{Stationary axi-symmetric state}

Equation (1) admits simple basic state, under the shape of stationary axi-symmetric solution. The mass conservation then implies:

$\frac{1}{r} \partial_{r}\left(r \Sigma u_{r}\right)=0$

$u_{r}=\frac{M_{t}}{\Sigma r}$

where $M_{t}$ is a constant, dimensionally equivalent to an accretion rate. Plugging this into the radial and azimuthal component of Eq. (1), we obtain two equations:

$$
\begin{aligned}
& -\frac{M_{t}^{2}}{\Sigma r^{3}}\left(1+\frac{r \partial_{r} \Sigma}{\Sigma}\right)-\frac{\Sigma u_{\theta}^{2}}{r}=-\partial_{r} H p-\Sigma \frac{G M}{r^{2}} \\
& \quad+\frac{2 \mu M_{t}}{\Sigma r^{3}}\left(-\frac{r \partial_{r} \mu}{\mu}\left(1+\frac{r \partial_{r} \Sigma}{\Sigma}\right)-\frac{r^{2} \partial_{r}^{2} \Sigma}{\Sigma}+2 \frac{\left(r \partial_{r} \Sigma\right)^{2}}{\Sigma^{2}}-\frac{r \partial_{r} \Sigma}{\Sigma}\right) \\
& \frac{M_{t}}{r^{2}} \partial_{r}\left(r u_{\theta}\right)=\frac{1}{r^{2}} \partial_{r}\left(\mu r^{3} \partial_{r} \frac{u_{\theta}}{r}\right) .
\end{aligned}
$$

The general solution of the second equation of (7) is:

$u_{\theta}=A \exp \left(\int_{r_{\mathrm{i}}}^{r}(\beta+1) \mathrm{d} x / x\right)+\frac{B}{r}$,

where $A$ and $B$ are constants and $\beta=M_{t} / \mu$. Plugging this solution into the first equation of (7) then defines the general pressure.

The basic state depends on three constants $A, B$ and $M_{t}$, which must be specified through some sort of boundary conditions. In the case of astrophysical flows, the boundary conditions are not very well known and it is less easy to constrain the parameters. The condition that the rotation velocity of the disk matches the star velocity at the interaction radius only provides one relation between the three parameters:

$A+\frac{B}{r_{\mathrm{i}}}=r_{\mathrm{i}} \Omega_{*}$.

An additional constraint comes from the hypothesis that the disk is geometrically thin. This is consistent with our assumption that the Mach number is less than unity. In that case, as soon as there is no dramatic variation of the thermodynamic variables, radial pressure gradients and terms involving the radial velocity ${ }^{1}$ can be neglected compared to the gravity, and the only way to satisfy the first equation of (7) is to set $M_{t} / \mu=\beta=-3 / 2$. In this case, the disk is almost Keplerian and obeys:

$u_{r}=-\frac{3 \mu}{2 \Sigma r}$

$u_{\theta}=\frac{\sqrt{G M}}{r^{1 / 2}}+\frac{r_{\mathrm{i}}^{2}}{r}\left(\Omega_{*}-\Omega_{K}\left(r_{\mathrm{i}}\right)\right)$,

1 The viscosity and the advection terms are then negligible compared to the radial pressure gradient by a factor of the order of $M a \frac{H}{r}$ and $M a^{2}$, respectively. 


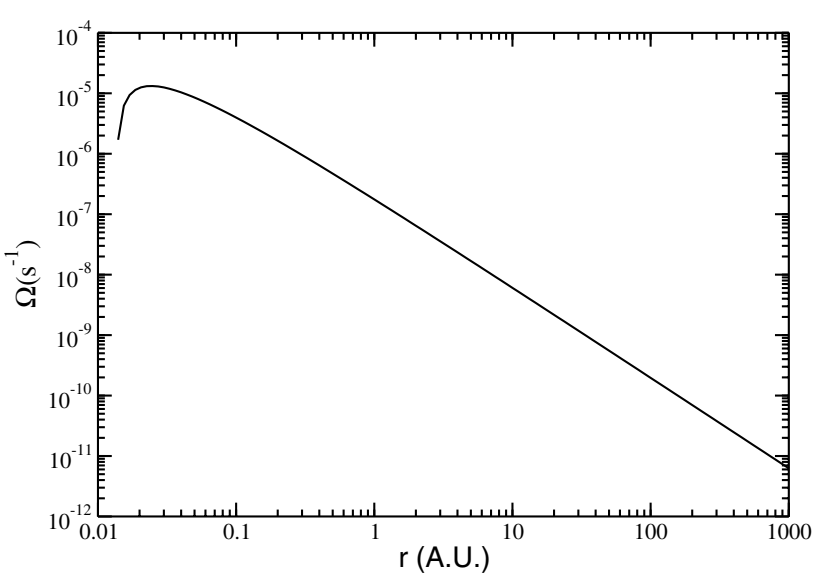

Fig. 2. Velocity profile in a circumstellar disk in the viscous regime, with Keplerian velocity in the outer part, and smooth matching onto the central object at the interaction radius. For this example, the mass of the central star has been taken as a solar mass.

where $\Omega_{K}\left(r_{\mathrm{i}}\right)$ is the angular Keplerian velocity at $r=r_{\mathrm{i}}$. The corresponding solution is plotted in Fig. 2. It is made of a Keplerian disk in the outer part, with a continuous matching towards the star velocity at the interaction radius.

\subsection{Comment about the disk outer radius}

In the expression we derived for the velocity, we did not need to specify anything about the condition at the outer boundary of the disk. One may then wonder what determines this boundary, and whether it is relevant in specifying the geometry of the problem. One way to answer this question is to note that stationary solutions of the shape (10) are only possible provided the dynamical time scales are short with respect to the viscous time scale. In the vertical direction, the dynamical time scale to ensure hydrostatic equilibrium is $H / c_{s} \sim \Omega_{K}^{-1}$. In the horizontal plane, the two dynamical time scale are the radial time scale $r / u_{r} \sim r^{2} \Sigma / \mu$ and the orbital time scale $\Omega^{-1} \sim \Omega_{K}^{-1}$. The radial time scale is comparable to the viscous time scale. So the condition for stationarity is that the orbital time scale is less than the viscous time scale, resulting in $r<r_{\mathrm{o}}$, with $r_{\mathrm{o}}$ solution of the equation:

$r_{\mathrm{o}}=\left.\left(\frac{\mu}{\Sigma \Omega}\right)^{1 / 2}\right|_{r=r_{\mathrm{o}}}$.

This radius defines the outer geometrical limit within which stationary solutions can reasonably exist. If in this formula one considers the ordinary viscosity, then one typically obtains $r_{\mathrm{o}}$ much larger than the observed disk outer radii. On the other hand, one may argue that as soon as the disk is turbulent, the molecular viscosity becomes irrelevant and one must consider a kind of "turbulent viscosity" in this formula. In this case, using the formula we derive in Sect. 3.3, we find that $r_{\mathrm{o}}$ is of the order of the disk scale height. In geometrically thin disks, it is not clear whether this limit really exists or not. Neither limits really match the observed disk radii. However, the sharp observed edge of disks remains inconsistent with stationary models. Stationary solutions, due to constant accretion rate in radius, are indeed in essence radially infinite. This suggests that the disk outer radii may still be linked to some non-stationary effects. This is the subject of a forthcoming paper (Mayer et al. 2004 , in preparation).

\subsection{The incompressible analog}

Astrophysical disks are (weakly) compressible and radially stratified. It is however possible to build an incompressible ana$\log$ of them, using clever boundary conditions. This remark is at the heart of the laboratory prototype. Consider an incompressible, unstratified fluid, enclosed within a domain with cylindrical symmetry, bounded by inner and outer radii $r_{\mathrm{i}}$ and $r_{\mathrm{o}}$. Its equations of motion are given by the Navier-Stokes equations:

$\partial_{t} \boldsymbol{u}+\boldsymbol{u} \cdot \nabla \boldsymbol{u}=-\frac{1}{\rho} \nabla p+v \Delta \boldsymbol{u}$

$\nabla \cdot \boldsymbol{u}=0$,

where $\rho$ is the density, $\boldsymbol{u}$ is the velocity, $v$ the molecular viscosity and $p$ is the pressure. If we assume hydrostatic equilibrium in the vertical direction, we get:

$\partial_{z} p \simeq 0$,

so that $p$ is a function of $r$ only. In that case, Eq. (12) admits the basic state of stationary solutions, with axial and translational symmetry along the disk rotation axis (the velocity only depends on $r$ ). The incompressibility condition then implies:

$\frac{1}{r} \partial_{r}\left(r u_{r}\right)=0$,

or

$u_{r}=\frac{K}{r}$

where $K$ is a constant, to be constrained later. Plugging this into the radial and azimuthal component of Eq. (12), we obtain two equations:

$$
\begin{aligned}
-\frac{K^{2}}{r^{3}}-\frac{u_{\theta}^{2}}{r} & =-\partial_{r} \frac{\Pi}{\rho}, \\
\frac{K}{r^{2}} \partial_{r}\left(r u_{\theta}\right) & =\frac{v}{r^{2}} \partial_{r}\left(r^{3} \partial_{r} \frac{u_{\theta}}{r}\right) .
\end{aligned}
$$

The second equation of Eq. (16) is homogeneous in $r$. It only admits two power law solutions, with exponent -1 and $1+K / v$, so that the general solution is:

$u_{\theta}=A r^{1+\beta}+\frac{B}{r}$,

where $A$ and $B$ are constants and $\beta=K / v$. Plugging this solution into the first equation of (16) then defines the pressure.

The basic state depends on three constants $A, B$ and $K$, which must be specified through boundary conditions. In laboratory flows, these conditions are usually well defined and allow for a simple determination of the constants once the rotation velocities at the inner and outer boundaries are known (Bahl 1970):

$A=\frac{r_{\mathrm{o}}^{-\beta}}{1-\eta^{\beta+2}}\left(\Omega_{o}-\eta^{2} \Omega_{i}\right)$,
$B=\frac{r_{\mathrm{i}}^{2}}{1-\eta^{\beta+2}}\left(\Omega_{i}-\Omega_{o} \eta^{\beta}\right)$, 
where $\eta=r_{\mathrm{i}} / r_{\mathrm{o}}$ is the radius ratio, $\Omega_{\mathrm{o}}$ and $\Omega_{\mathrm{i}}$ are the angular velocity at outer and inner radii and $\beta=R_{r}=K / v=u_{r}\left(r_{\mathrm{i}}\right) r_{\mathrm{i}} / v$ is the radial Reynolds number, based on the radial velocity through the wall of the inner cylinder. Note that it is positive for motions outwards from the axis of rotation.

Comparing Eq. (10) with Eqs. (17) and (18), it is possible to see that the "laboratory" analog of Keplerian flow is such that:

$\beta=-\frac{3}{2}$,

$\Omega_{i}=\Omega_{*}$,

$\Omega_{\mathrm{o}}=\sqrt{\frac{G M}{r_{\mathrm{o}}^{3}}}$,

$\eta=r_{\mathrm{i}} / r_{\mathrm{o}}$.

This shows that this basic state also describes laboratory incompressible flows, with rigid boundaries and without gravity, in which angular momentum distribution may be imposed by boundary conditions, and astrophysical flows, without rigid boundaries, in which angular momentum distribution is imposed by gravity. In other words, building a prototype of astrophysical disks (within the approximations described above) requires a (laboratory) flow with equivalent angular momentum distribution, and equivalent control parameters. We now derive these control parameters, in order to apply the Reynolds similarity principle.

\subsection{Control parameters}

The shape of the basic state allows for the determination of the control parameters of the flow. These parameters are essential in the comparison with the laboratory prototype since the Reynolds similarity principle states that the astrophysical disk will behave like the laboratory prototype with same control parameters. These control parameters are

the global Reynolds number:

$\operatorname{Re}=\frac{\bar{S}\left(r_{\mathrm{o}}-r_{\mathrm{i}}\right)^{2}}{v}$,

the rotation number:

$R_{\Omega}=\frac{2 \bar{\Omega}}{\bar{S}}$

the curvature number

$R_{\mathrm{C}}=\frac{\bar{r}}{r_{\mathrm{o}}-r_{\mathrm{i}}}$,

the local radial Reynolds number:

$R_{r}=\frac{u_{r} r}{v}$

The aspect ratio:

$\Gamma=\frac{\bar{H}}{\bar{r}}$.

Here, $\bar{\Omega}, \bar{S}$ and $\bar{r}$ are the characteristic angular velocity, shear and radius. Adopting the convention of Dubrulle et al. (2004), we find:

$\bar{\Omega}=\Omega_{K}\left(r_{\mathrm{i}}\right)\left(\frac{r_{\mathrm{i}}}{r_{\mathrm{o}}}\right)^{1 / 2}+\frac{r_{\mathrm{i}}}{r_{\mathrm{o}}}\left(\Omega_{*}-\Omega_{K}\left(r_{\mathrm{i}}\right)\right)$
$\bar{S}=\frac{1}{2} \Omega_{K}\left(r_{\mathrm{i}}\right)\left(\frac{r_{\mathrm{i}}}{\bar{r}}\right)^{3 / 2}-2 \bar{\Omega}$,

while $\bar{r}$ is fixed through the condition $\bar{\Omega}=u_{\theta}(\bar{r}) / \bar{r}$. A simplification occurs in two limiting cases, relevant to astrophysical disks: $r_{\mathrm{i}} \ll r_{\mathrm{o}}$ or $r_{\mathrm{i}} \rightarrow r_{\mathrm{o}}, \quad \Omega_{*} \rightarrow \Omega_{K}\left(r_{\mathrm{i}}\right)$. In both cases, we have:

$$
\begin{aligned}
\bar{r} & =r_{\mathrm{i}}^{2 / 3} r_{\mathrm{o}}^{1 / 3}, \\
\bar{\Omega} & =\Omega_{K}(\bar{r}), \\
\bar{S} & =-\frac{3}{2} \Omega_{K}(\bar{r}) .
\end{aligned}
$$

The control parameters then simplify into:

$$
\begin{aligned}
& \operatorname{Re}=\frac{3}{2} \frac{\Omega_{K}(\bar{r})\left(r_{\mathrm{o}}-r_{\mathrm{i}}\right)^{2}}{v}, \\
& R_{\Omega}=-\frac{4}{3}, \\
& R_{\mathrm{C}}=\frac{r_{\mathrm{i}}^{2 / 3} r_{\mathrm{o}}^{1 / 3}}{r_{\mathrm{o}}-r_{\mathrm{i}}}, \\
& R_{r}=-\frac{3}{2}, \\
& \Gamma=\frac{H}{r_{\mathrm{i}}^{2 / 3} r_{\mathrm{o}}^{1 / 3}} .
\end{aligned}
$$

If the disk is stratified or magnetized, other control parameters appear, like the Prandtl number $\operatorname{Pr}=\nu / \kappa$ and the magnetic Prandtl number $\mathrm{Pm}=\mu_{0} v / \eta$ where $\kappa$ and $\eta$ are the heat diffusivity and the magnetic resistivity, and $\mu_{0}$ is the vacuum permeability.

\subsection{Molecular transport processes}

The computation of the control parameters requires an estimate of molecular transport coefficients. These coefficients depend on the ionization state of the gas. There are two sources of ionization. Thermal ionization is efficient in the inner part of the disk. The corresponding ionization fraction can be written as (Fromang et al. 2003):

$$
\begin{aligned}
x_{e}^{t h}= & 6 \times 10^{-2}\left(\frac{T}{1000 \mathrm{~K}}\right)^{3 / 4}\left(\frac{2.4 \times 10^{15} \mathrm{~cm}^{-3}}{n}\right)^{1 / 2} \\
& \times \exp (-25188 \mathrm{~K} / T),
\end{aligned}
$$

where $T$ and $n$ are the temperature and the number density of neutral species (hydrogen mainly), respectively. For temperature lower than $10^{3} \mathrm{~K}$ (typically $r>1 \mathrm{AU}$ ), thermal ionization is negligible. However, X-ray illumination from the central star (or the magnetospheric accretion flow) may induce a weak ionization in some part of the disk (typically away from the midplane) (Feigelsson \& Montmerle 1999). A theoretical study has been performed by Igea \& Glassgold (1999). They found that at a given radius from the source, the ionization fraction is a universal function of the vertical column density $N_{\perp}$, independent of the structural details of the disk. The role of cosmic 
rays in the disk ionization is still a matter of debate (Sano et al. 2000) and will not be discussed here. For a typical young stellar object, Igea and Glassgold's result can be approximated by:

$$
\begin{gathered}
x_{\mathrm{e}}^{X}=\frac{n_{\mathrm{e}}}{n}=\frac{10^{17} \mathrm{~cm}^{-2}}{N_{\perp}} T^{1 / 4} n^{-1 / 2} \mathrm{e}^{-0.002\left(r_{\mathrm{AU}}-1\right)}, \\
N_{\perp}>10^{20} \mathrm{~cm}^{-2}, \\
x_{\mathrm{e}}=\frac{n_{\mathrm{e}}}{n}=10^{-3} T^{1 / 4} n^{-1 / 2} \mathrm{e}^{-0.002\left(r_{\mathrm{AU}}-1\right)}, \\
N_{\perp}<10^{20} \mathrm{~cm}^{-2},
\end{gathered}
$$

where $n_{\mathrm{e}}$ is the ion number density, and $r_{\mathrm{AU}}$ is the distance from the central star, in astronomical units.

When the gas is neutral, the viscosity and heat diffusivity are given by (Lang 1980):

$v_{\text {neu }}=\kappa=3 \times 10^{19} \frac{T^{1 / 2}}{n} \mathrm{~cm}^{2} \mathrm{~s}^{-1}$,

where $T$ is the disk temperature, and $n$ the number density.

When the gas is weakly ionized $\left(x_{\mathrm{e}} \ll 1\right)$, the transport coefficient must be multiplied by a factor (Lang 1980):

$\frac{v_{\text {ion }}}{v_{\text {neu }}}=4 \times 10^{-12} \frac{T^{2}}{x_{\mathrm{e}}}$.

This correction is valid as long as $v_{\text {ion }} / v_{\text {neu }}<1$. This sets a limit on ionization fraction, below which the gas viscosity takes the neutral value:

$x_{\mathrm{e}}^{\mathrm{cr}}=4 \times 10^{-12} T^{2}$.

The Prandtl number in this case is equal to $10^{-11}$ (Lang 1980).

The resistivity of an ionized gas can be written as the sum of the resistivity induced by electron-neutral collisions and electron-ion collisions:

$\eta=\eta_{\mathrm{en}}+\eta_{\mathrm{ei}}$

where (Lang 1980)

$\eta_{\mathrm{en}}=10^{-6} \frac{1-x_{\mathrm{e}}}{x_{\mathrm{e}}} T^{1 / 2} \mathrm{ohm}-\mathrm{cm}$

$\eta_{\mathrm{ei}}=4 \times 10^{3} \frac{\ln \Lambda}{T^{3 / 2}} \mathrm{ohm}-\mathrm{cm}$

where $\Lambda=1.3 \times 10^{4} \frac{T^{3 / 2}}{N_{\mathrm{e}}^{1 / 2}}$ is the Coulomb logarithm.

\subsection{Physical parameters}

Various physical parameters are required to estimate the control parameter.

Parameters associated with the disk are $r_{\mathrm{o}}, r_{\mathrm{i}}, \Gamma$ and $v$. The disk inner radius depends on whether the disk/star interaction is direct or indirect. In the first case, $r_{\mathrm{i}}=r_{*}$. In the second case, $r_{\mathrm{i}}$ may not exceed the corotation radius at which the disk velocity matches the star velocity. In the following, we will consider variations of $r_{\mathrm{i}}$ in between these two limits. The disk outer radius $r_{\mathrm{o}}$ must be specified through the implicit relation (11). Direct observations of the disk suggest that the disk size is of the order of $r_{\mathrm{D}}=1000 \mathrm{AU}$ for the disk around T Tauri and even smaller, $r_{\mathrm{D}}=50 \mathrm{AU}$, for the disk around FU ORI
(Kenyon 1999). Clearly, $r_{\mathrm{D}}$ is thus the maximum size $r_{\mathrm{o}}$ can achieve. For practical reasons, we defer its discussion to the next section, after computation of the temperature and density profile.

Parameters relative to the proto-star have been measured in some T Tauri and FU Ori stars. Table 1 gives the sample of stars we shall use in the following. It is particularly convenient and illustrative to scale all quantities in the problem with respect to values defined at the distance of $\bar{r}=0.33 \mathrm{AU}$, which is the characteristic radius corresponding to a disk with $r_{\mathrm{i}}=10^{11} \mathrm{~cm}$ and $r_{\mathrm{o}}=1000 \mathrm{AU}$, the two extreme limits for $r_{\mathrm{i}}$ and $r_{\mathrm{o}}$. We also use, as a reference, a rotation period of the star of 8 days (typical T Tauri star), leading to $\Omega_{*}=9 \times 10^{-6} \mathrm{~s}^{-1}$.

The temperature and number density in circumstellar disks are not known due to the lack of spatial resolution. However, their magnitude can be deduced by short radii extrapolation of measurements made on the outer disk. The inversion method of Guilloteau \& Dutrey (1998), based on $\chi$-square fitting of CO interferometric maps, yields temperature and density profiles at $r \gtrsim 50-100$ AU. For instance, for the disk around DM Tau $\left(M \simeq 0.5 M_{\odot}\right)$, their method predicts

$$
\begin{cases}T \simeq 30\left(\frac{r}{100 \mathrm{AU}}\right)^{-0.6} & \mathrm{~K} \\ n \simeq 10^{8}\left(\frac{r}{100 \mathrm{AU}}\right)^{-2.75} & \mathrm{~cm}^{-3} \\ \Sigma \simeq 1\left(\frac{r}{100 \mathrm{AU}}\right)^{-1.5} & \mathrm{~g} \mathrm{~cm}^{-2}\end{cases}
$$

where $\Sigma$ is the surface density of the disk (including gas and dust). Error bars on the measurements are rather large, and could amount to a possible variation by a factor of 5 to 10 . At $r=1 \mathrm{AU}$, one finds $n \simeq 3 \times 10^{13} \mathrm{~cm}^{-3}$ and $\Sigma \simeq 1000 \mathrm{~g} \mathrm{~cm}^{-2}$, in agreement with values obtained by modeling the deuterium enrichment in the Solar System (Drouart et al. 1999; Hersant et al. 2001). At the reference radius of $0.33 \mathrm{AU}$, the density is $\bar{n} \simeq 7 \times 10^{14} \mathrm{~cm}^{-3}$, the surface density is $\bar{\Sigma} \simeq 5275 \mathrm{~g} \mathrm{~cm}^{-2}$, the height is $\bar{H}=4.6 \times 10^{12} \mathrm{~cm}$ and the temperature is $\simeq 925 \mathrm{~K}$. In a more recent analysis of the disk around BP Tau, Dutrey et al. (2003) found densities and temperature corresponding to a value of $\bar{n} \simeq 3 \times 10^{14} \mathrm{~cm}^{-3}, \bar{\Sigma} \simeq 992 \mathrm{~g} \mathrm{~cm}^{-2}, \bar{H}=10^{11} \mathrm{~cm}$ and $\bar{T}=289 \mathrm{~K}$. The difference between these figures and the figures of DM Tau provide an illustration of the error bars associated with our "typical values", since the disk around BP Tau seems much smaller, and corresponds to a more evolved stage than the disk around DM Tau.

\subsubsection{Ionization state}

It is interesting to study the ionization state of the disk with temperature and density observed in DM Tau. The ionization fraction is plotted as a function of radius in Fig. 3 for the thermal and X-ray contribution. One sees that the thermal contribution dominates at $r<1 \mathrm{AU}$, while the X-ray contribution becomes important at larger radii. However, comparing with the limiting ionization state Eq. (33), one sees that only the outer part of the disk $r>100 \mathrm{AU}$ is sufficiently ionized to influence the molecular viscosity. 
Table 1. Observational parameters for T Tauri stars (from Bouvier 1990; Hartmann et al. 1998) and for FU Ori stars (from Popham et al. 1996) considered in this study (left) and disk physical parameters (right). The computation of the corotation radius requires the knowledge of the stellar rotation velocity. In case this last quantity is not available, the corotation radius has been set to $10.05 R_{\odot}$, the solar value. Lower and upper bound on $\dot{M}_{0}$ and Re have been computed using either the star radius or the corotation radius for $r_{\mathrm{i}}$. Accretion rates are in $M_{\odot} \mathrm{yr}^{-1}$.

\begin{tabular}{|c|c|c|c|c|c|c|c|c|c|}
\hline \multirow[b]{3}{*}{ Star } & \multicolumn{5}{|c|}{ input parameters } & \multicolumn{4}{|c|}{ output parameters } \\
\hline & \multirow{2}{*}{$\begin{array}{c}\text { Mass } \\
M / M_{\odot}\end{array}$} & \multirow{2}{*}{$\begin{array}{c}\text { Radius } \\
r_{*} / R_{\odot} \\
\end{array}$} & \multirow{2}{*}{$\begin{array}{c}\text { Coro. radius } \\
r_{\text {coro }} / R_{\odot}\end{array}$} & \multirow{2}{*}{$\begin{array}{c}\text { Disk outer edge } \\
r_{\mathrm{o}} / 1000 \mathrm{AU}\end{array}$} & \multirow{2}{*}{$\begin{array}{c}\text { Accr. rate } \\
\log \dot{M}\end{array}$} & \multicolumn{2}{|c|}{ Eff. rates } & \multicolumn{2}{|c|}{ Reynolds num. } \\
\hline & & & & & & $\log \dot{M}_{0}\left(r_{*}\right)$ & $\log \dot{M}_{0}\left(r_{\text {coro }}\right)$ & $\log \mathrm{Re}_{*}$ & $\log \operatorname{Re}_{\text {coro }}$ \\
\hline AA Tau & 0.53 & 1.74 & 13.81 & 1 & -8.48 & -4.39 & -3.67 & 13.53 & 12.55 \\
\hline BP Tau & 1.32 & 1.99 & 17.79 & 1 & -7.54 & -2.87 & -2.10 & 13.27 & 12.24 \\
\hline CY Tau & 0.42 & 1.63 & 10.05 & 1 & -8.12 & -4.17 & -3.54 & 13.61 & 12.74 \\
\hline DE Tau & 0.25 & 2.45 & 10.05 & 1 & -7.58 & -4.00 & -3.52 & 13.54 & 12.85 \\
\hline DF Tau & 0.27 & 3.37 & 11.29 & 1 & -6.91 & -3.31 & -2.90 & 13.39 & 12.78 \\
\hline DK Tau & 0.43 & 2.49 & 10.05 & 1 & -7.42 & -3.49 & -3.01 & 13.42 & 12.73 \\
\hline DN Tau & 0.38 & 2.09 & 10.03 & 1 & -8.46 & -4.60 & -4.06 & 13.52 & 12.76 \\
\hline DO Tau & 0.37 & 2.25 & 10.05 & 1 & -6.84 & -3.00 & -2.48 & 13.50 & 12.77 \\
\hline DQ Tau & 0.44 & 1.79 & 10.05 & 1 & -9.40 & -5.43 & -4.83 & 13.56 & 12.73 \\
\hline DS Tau & 0.87 & 1.36 & 10.05 & 1 & -7.89 & -3.45 & -2.76 & 13.53 & 12.58 \\
\hline GG Tau & 0.44 & 2.31 & 10.05 & 1 & -7.76 & -3.81 & -3.30 & 13.45 & 12.73 \\
\hline GI Tau & 0.71 & 1.48 & 10.05 & 1 & -8.02 & -3.72 & -3.06 & 13.53 & 12.63 \\
\hline GK Tau & 0.46 & 2.15 & 10.05 & 1 & -8.19 & -4.21 & -3.67 & 13.47 & 12.72 \\
\hline GM Aur & 0.52 & 1.78 & 10.05 & 1 & -8.02 & -3.95 & -3.34 & 13.52 & 12.69 \\
\hline HN Tau & 0.81 & 0.76 & 10.05 & 1 & -8.89 & -4.45 & -3.55 & 13.80 & 12.60 \\
\hline IP Tau & 0.52 & 1.44 & 10.05 & 1 & -9.10 & -5.00 & -4.33 & 13.62 & 12.69 \\
\hline UY Tau & 0.42 & 2.60 & 10.05 & 1 & -7.18 & -3.27 & -2.80 & 13.41 & 12.74 \\
\hline CI Tau & 0.5 & 1.87 & 10.05 & 1 & -7.19 & -3.14 & -2.56 & 13.51 & 12.70 \\
\hline CX Tau & 0.33 & 1.63 & 10.05 & 1 & -8.97 & -5.18 & -4.55 & 13.66 & 12.79 \\
\hline CZ Tau & 0.41 & 1.19 & 10.05 & 1 & -9.35 & -5.39 & -4.65 & 13.75 & 12.74 \\
\hline DM Tau & 0.43 & 1.39 & 10.05 & 1 & -7.95 & -3.97 & -3.29 & 13.67 & 12.73 \\
\hline DD Tau & 0.42 & 1.44 & 10.05 & 1 & -8.39 & -4.43 & -3.76 & 13.66 & 12.74 \\
\hline DH Tau & 0.38 & 1.67 & 11.33 & 1 & -8.30 & -4.42 & -3.76 & 13.62 & 12.71 \\
\hline DI Tau & 0.43 & 1.71 & 12.56 & 1 & -8.75 & -4.79 & -4.01 & 13.58 & 12.64 \\
\hline DP Tau & 0.46 & 1.44 & 10.05 & 1 & -7.88 & -3.86 & -3.19 & 13.64 & 12.72 \\
\hline FM Tau & 0.58 & 1.17 & 10.05 & 1 & -8.45 & -4.26 & -3.52 & 13.68 & 12.67 \\
\hline FO Tau & 0.33 & 1.59 & 10.05 & 1 & -7.50 & -3.71 & -3.07 & 13.67 & 12.79 \\
\hline FQ Tau & 0.35 & 1.42 & 10.05 & 1 & -6.45 & -2.61 & -1.93 & 13.71 & 12.78 \\
\hline FS Tau & 0.46 & 1.25 & 10.05 & 1 & -8.09 & -4.06 & -3.34 & 13.70 & 12.72 \\
\hline FV Tau & 0.71 & 1.87 & 10.05 & 1 & -6.23 & -1.95 & -1.37 & 13.44 & 12.63 \\
\hline FX Tau & 0.34 & 1.94 & 10.05 & 1 & -8.65 & -4.86 & -4.28 & 13.58 & 12.79 \\
\hline FY Tau & 0.50 & 1.87 & 10.05 & 1 & -7.41 & -3.36 & -2.78 & 13.51 & 12.70 \\
\hline GH Tau & 0.29 & 1.90 & 10.05 & 1 & -7.92 & -4.23 & -3.65 & 13.62 & 12.82 \\
\hline GO Tau & 0.50 & 1.40 & 10.05 & 1 & -7.93 & -3.86 & -3.17 & 13.64 & 12.70 \\
\hline Haro 6-37 & 0.60 & 1.90 & 10.05 & 1 & -7.00 & -2.83 & -2.26 & 13.46 & 12.66 \\
\hline HO Tau & 0.56 & 0.94 & 10.05 & 1 & -8.86 & -4.68 & -3.86 & 13.79 & 12.68 \\
\hline IQ Tau & 0.35 & 2.01 & 10.05 & 1 & -7.55 & -3.74 & -3.18 & 13.56 & 12.78 \\
\hline $\mathrm{LkCa} 15$ & 0.81 & 1.53 & 10.05 & 1 & -8.87 & -4.49 & -3.84 & 13.49 & 12.60 \\
\hline Lk Ha 332/G1 & 0.29 & 2.36 & 10.05 & 1 & -6.60 & -2.93 & -2.43 & 13.53 & 12.82 \\
\hline V955 Tau & 0.44 & 2.34 & 10.05 & 1 & -7.02 & -3.07 & -2.57 & 13.44 & 12.73 \\
\hline V1057 Cygni & 0.50 & 1.60 & 13.53 & 0.005 & -4.00 & -0.86 & -0.12 & 14.73 & 13.72 \\
\hline FU Ori & 0.70 & 1.20 & 15.83 & 0.005 & -3.70 & -0.31 & -0.58 & 14.78 & 13.58 \\
\hline
\end{tabular}

\subsection{Regimes}

The previous scaling allows for an estimate of the disk control parameter, and, therefore, for an identification of the possible regimes. A difficulty with respect to the laboratory experiment is that in disks, the transport coefficients vary over across the disk due to the radial stratification. To define the control parameter, one must pick a typical value. In this context, it is logical to consider their value at $r=\bar{r}$, since both the typical shear and 


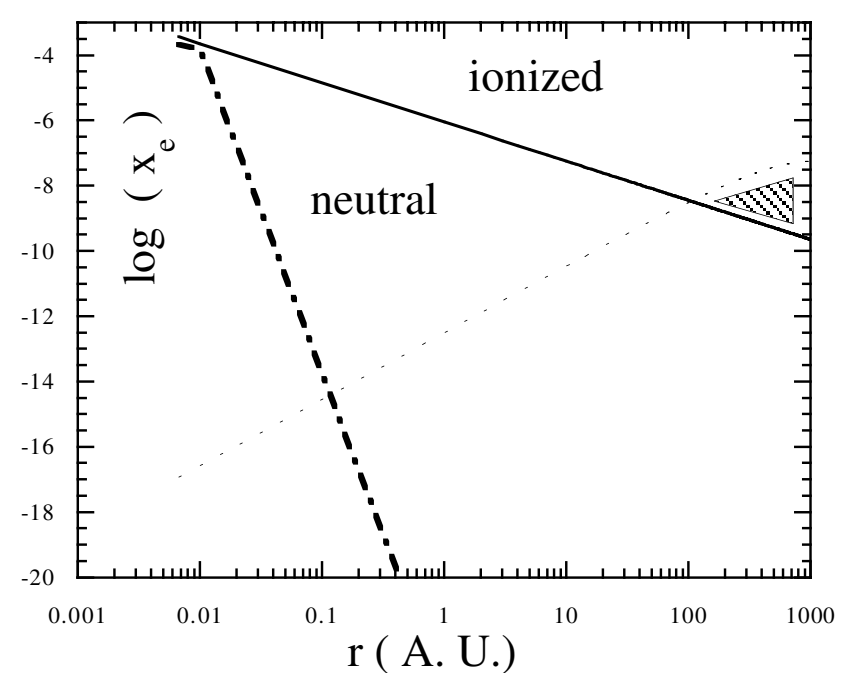

Fig. 3. Midplane ionization fraction due to thermal contribution (dotdashed line) and X-ray contribution (dot-dotted line). The solid line is the limiting ionization fraction, below which the ionization does not influence molecular transport. The shaded area is the region where ionization has to be taken into account in the computation of the viscosity.

radii at this location have been used. Using the values given in the previous section, we then obtain:

$$
\begin{aligned}
\operatorname{Re}= & 2 \times 10^{25}\left(\frac{M}{M_{\odot}}\right)^{1 / 2}\left(\frac{\bar{n}}{7 \times 10^{14} \mathrm{~cm}^{-3}}\right)\left(\frac{\bar{T}}{930 \mathrm{~K}}\right)^{-1 / 2} \\
& \times\left(\frac{r_{\mathrm{i}}}{10^{11} \mathrm{~cm}}\right)^{-4 / 3}\left(\frac{r_{\mathrm{o}}}{10^{3} \mathrm{AU}}\right)^{-2 / 3} \\
R_{\Omega}= & -\frac{4}{3} \\
R_{r}= & -\frac{3}{2} \\
\operatorname{Pr}= & 1 \\
\operatorname{Pm}= & 2 \times 10^{-8} \\
\Gamma= & 0.94 .
\end{aligned}
$$

The value of the rotation number indicates that the flow is anticyclonic and belongs to the "globally sub-critical" class defined in Dubrulle et al. (2004). The radial Reynolds number is negative, indicating an inward radial circulation. Its value is close to unity. So its influence on transport properties can be neglected as a first approximation, see Dubrulle et al. (2004). The curvature number depends on the interpretation of the viscous time scale (see Sect. 2.9.2). In the case where the interpretation is done with the molecular viscosity, one finds $R_{\mathrm{C}}=0.0004$, that is, disks are in the wide gap limit. In the other limiting case where the viscous time scale is computed using the turbulent viscosity, one finds $R_{\mathrm{C}}=1-\Gamma$, that is, disks are in the small gap limit. The relevant Reynolds parameter to be considered in studying transport properties will be $\operatorname{Re} R_{\mathrm{C}}^{2}=\bar{S} \bar{r}^{2} / v$, see Dubrulle et al. (2004). Finally, the aspect ratio is less than one. From the review of Dubrulle et al, we infer that an additional correction $\Gamma^{2}$ must be included in the definition of the relevant
Reynolds parameter, which becomes:

$$
\begin{aligned}
\operatorname{Re}_{\text {phys }}= & \operatorname{Re}\left(R_{\mathrm{C}} \Gamma\right)^{2}=\frac{\bar{S} \bar{H}^{2}}{v} \\
= & 3 \times 10^{13}\left(\frac{M_{*}}{M_{\odot}}\right)^{-1 / 2}\left(\frac{\bar{n}}{7 \times 10^{14} \mathrm{~cm}^{-3}}\right) \\
& \times\left(\frac{\bar{T}}{930 \mathrm{~K}}\right)^{1 / 2}\left(\frac{r_{\mathrm{in}}}{10^{11} \mathrm{~cm}}\right)^{-1}\left(\frac{r_{\mathrm{o}}}{10^{3} \mathrm{AU}}\right)^{-1 / 2} .
\end{aligned}
$$

This is the expression one would naturally derive by considering the "smallest" length scale in the problem, see e.g. Longaretti (2003).

\section{Predictions about the structure of circumstellar disks}

\subsection{Stability: The laminar/turbulent transition}

The stability properties of circumstellar disks can be found by comparing the physical Reynolds number $\mathrm{Re}_{\text {phys }}$ with critical Reynolds numbers derived in laboratory experiments, in the anti-cyclonic non-linear regime. These measurements are summarized in Dubrulle et al. (2004). Disregarding any body forces, one finds a critical Reynolds number of the order of 2300 , well below the disk value. Taking into account the possible stable vertical stratification observed e.g. in DM Tau (Dartois et al. 2003) ${ }^{2}$, one obtains a slightly larger value of the order of 4000 (Dubrulle et al. 2005). The presence of a vertical magnetic field may increase the critical Reynolds number, due to the low magnetic Prandtl number prevailing in disks. Using the scaling of Willis \& Barenghi (2002), one finds a critical Reynolds number $\operatorname{Re}_{\mathrm{c}} \sim 100 / \mathrm{Pm}=10^{10}$. This is still well below the observed Reynolds number. These numbers lead us to conclude that the disk is turbulent.

However, due to the huge variation of the transport coefficients across the disk, one may wonder how strong this conclusion is. A way to answer this question is to see how locally in the disk, the stability criterion are satisfied using a "local" non-dimensionalized parameter, built by replacing $\bar{r}$ by $r$ the distance to the central object. The result of this procedure is plotted in Fig. 4. One sees that at any radius, such an effective local Reynolds number is well above any critical Reynolds number due to body forces. This strengthens our conclusion.

\subsection{Mean energy dissipation and accretion rate}

\subsubsection{Definition}

The quantitative comparison between experimental measurements and energy dissipated in circumstellar disks first requires a relation linking the torque and the disk luminosity $\mathcal{L}$. The total power dissipated in a Taylor-Couette experiment with same control parameter as in a Keplerian disk is

$\epsilon=\bar{v}^{2} \bar{\Sigma} \frac{\bar{S} G}{4}=\frac{G}{4 \operatorname{Re}_{\text {phys }}^{2}} \bar{\Sigma} \bar{S}^{3} \bar{H}^{4}$,

2 This may be due to disk illumination by the central star (D'Alessio et al. 1998). 


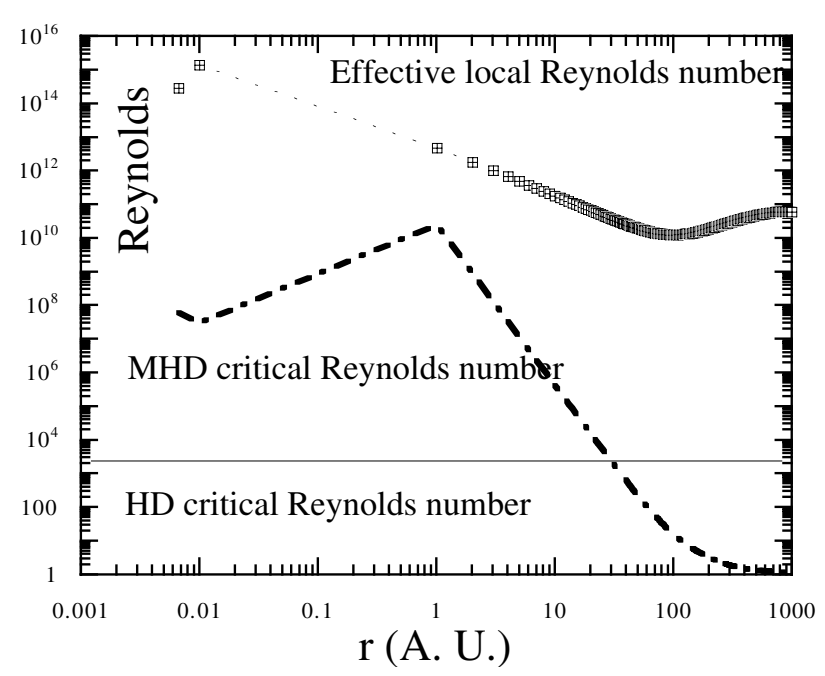

Fig. 4. Physical local Reynolds number in circumstellar disks as a function of radius (dotted-line with symbols). The dashed-dotted line and the full line are the critical Reynolds number deduced from laboratory experiments, see Dubrulle et al. (2004).

where $\bar{\Sigma}$ is the disk surface density, $G$ the non-dimensional torque and $\bar{v}$ the viscosity. In a stationary disk, this power is dissipated under the form of heat, and thus coincides with the disk luminosity, that is

$\mathcal{L}=\epsilon$.

In practice, observed luminosities are often expressed as a function of an "effective mass accretion rate", namely (Hartmann et al. 1998)

$\dot{M}=\frac{0.8 r_{*} \mathcal{L}}{\mathcal{G M}}$.

From a theoretical point of view, the detailed computation of the accretion luminosity is not straightforward since it depends on the boundary conditions at the interaction radius. For comparison with experimental data, we therefore consider the quantity:

$\frac{G}{\mathrm{Re}_{\text {phys }}^{2}}=\frac{\dot{M}}{\dot{M}_{0}}$,

where $\dot{M}_{0}$ is an effective accretion rate given by

$\dot{M}_{0}=\bar{\Sigma} r_{*} \bar{r} \bar{\Omega}\left(\frac{\bar{H}}{\bar{r}}\right)^{4}$.

The quantity $G / \operatorname{Re}_{\text {phys }}^{2}$ (the non-dimensional energy dissipation), includes all the boundary condition dependence and only depends on the Reynolds number.

\subsubsection{Prediction using laboratory experiments}

The results of Dubrulle et al. (2004) lead to an analytical prediction for the function $\dot{M} / \dot{M}_{0}=f\left(R_{\Omega}\right) G_{\mathrm{i}} / \mathrm{Re}_{\text {phys }}^{2}$, where $f\left(R_{\Omega}\right)$ is a function parameterizing the influence of rotation, and $G_{\mathrm{i}}$ is the torque in situation when only the inner cylinder is rotating. From the experiments, we infer $f\left(R_{\Omega}\right) \sim 0.1$ if the flow is turbulent, and 1 is the flow is laminar. Taking the wide gap limit $r_{\mathrm{i}} \ll r_{\mathrm{o}}, R_{\mathrm{C}}^{2} \mathrm{Re}=\mathrm{Re}_{\text {phys }}$ in the formulae of Dubrulle et al. (2004), we obtain three possible regimes:

- in the laminar regime, for $\mathrm{Re}_{\text {phys }} \leq 2300$

$$
\frac{\dot{M}}{\dot{M}_{0}}=\frac{2 \pi}{\mathrm{Re}_{\text {phys }}} \text {. }
$$

- for smooth boundary conditions and $\mathrm{Re}_{\text {phys }}>2300$

$$
\left.\frac{\dot{M}}{\dot{M}_{0}}\right|_{\text {smooth }}=0.06\left[\ln \left(3 \times 10^{-4} \mathrm{Re}_{\text {phys }}^{2}\right)\right]^{-3 / 2} \text {. }
$$

- for rough boundary conditions and $\mathrm{Re}_{\text {phys }}>2300$

$$
\left.\frac{\dot{M}}{\dot{M}_{0}}\right|_{\text {rough }}=1.9 \times 10^{-2} \text {. }
$$

In the other limit $r_{\mathrm{i}} \rightarrow r_{\mathrm{o}}$, the turbulent value follows a similar expression, but must be multiplied by a factor $1 / 3(1-\Gamma)^{3 / 2}$. In astrophysical disks, the boundary conditions are not known a priori. Moreover, given the huge physical difference between the inner part and the outer part, it is unlikely that the boundary conditions at the inner and outer part coincide, so that we are probably more in a state of "mixed" boundary conditions studied experimentally by Van den Berg et al. (2003). In that case, the energy dissipation is found to vary between the two limits set by respectively the "pure" smooth type (45) and the pure "rough" type (46), see Dubrulle et al. (2004). We shall therefore adopt these formulae as a lower and upper limit of the energy dissipation in disks.

\subsubsection{Test against observational data}

We find the following scaling for $\dot{M}_{0}$ :

$$
\begin{aligned}
\dot{M}_{0} \simeq & 3 \times 10^{-5}\left(\frac{\bar{\Sigma}}{5300 \mathrm{~g} \mathrm{~cm}^{-2}}\right)\left(\frac{M}{M_{\odot}}\right)^{-3 / 2}\left(\frac{r_{*}}{10^{11} \mathrm{~cm}}\right) \\
& \times\left(\frac{r_{\mathrm{i}}}{10^{11} \mathrm{~cm}}\right)^{-0.8}\left(\frac{r_{\mathrm{o}}}{10^{3} \mathrm{AU}}\right)^{-0.4}\left(\frac{\bar{T}}{930 \mathrm{~K}}\right)^{2} M_{\odot} / \mathrm{yr} .
\end{aligned}
$$

We are aware that these values are probably uncertain by a factor of 10 or even more. In the next decade, the results expected with ALMA will greatly reduce the error bars.

At $\operatorname{Re}=3 \times 10^{13}$, our model (Eqs. (45) and (46) predicts that $0.0002<\dot{M} / \dot{M}_{0}<0.019$, resulting in $6 \times 10^{-9}<\dot{M}<$ $6 \times 10^{-7} M_{\odot} \mathrm{yr}^{-1}$ for $\mathrm{T}$ Tauri stars. This is in good agreement with the observed values ranging from $\dot{M}=10^{-10}$ to $10^{-6} M_{\odot} \mathrm{yr}^{-1}$ (Hartmann et al. 1998). Disks around FU Ori are characterized by a smaller disk radius, leading to higher values of $\dot{M}$ according to Eq. (47), by a factor 5. This is not quite enough to reach values of up to $10^{-5} M_{\odot} \mathrm{yr}^{-1}$, associated with disks around FU Ori stars (Kenyon 1994). Such values could be obtained if the typical disk density is higher in disk around FU Ori, resulting in more massive disks. This is plausible, since FU Ori stars are younger than T-Tauri stars.

A graphical representation of this discussion can be obtained by plotting the computed $\dot{M} / \dot{M}_{0}$ as a function of $\mathrm{Re}_{\text {phys }}$ 


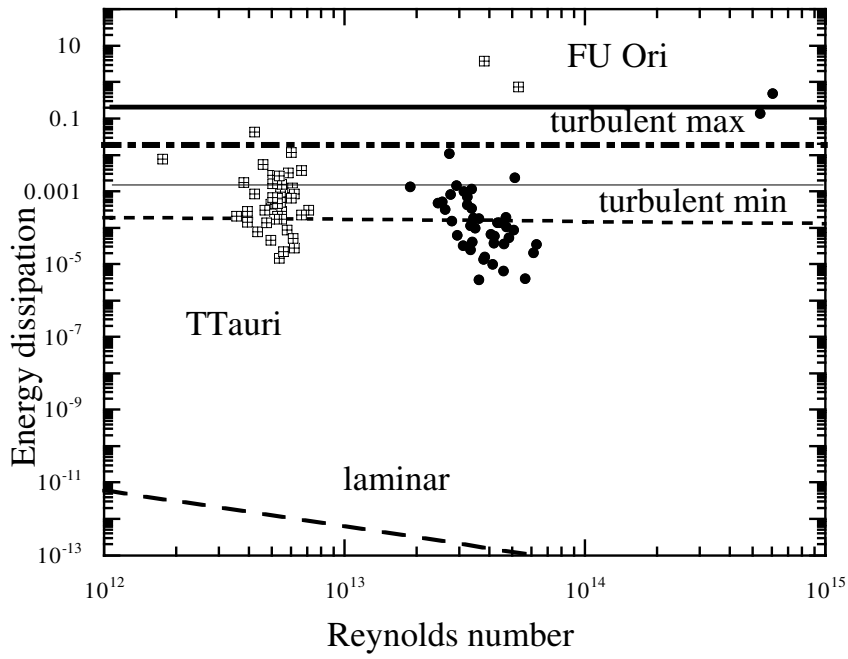

Fig. 5. Comparison between the non-dimensional energy dissipation predicted from laboratory measurements in the wide gap limit (dotted lines) or in the small gap limit (plain lines) and observed in circumstellar disks (symbols), as a function of the Reynolds number $\mathrm{Re}_{\text {phys }}$. For an easier comparison, the mean energy dissipation has been translated into the non-dimensional accretion rate $\dot{M} / \dot{M}_{0}$ (computed using Eq. (45) and observationally determined parameters reported in Table 1). The symbols $\boxplus$ report the value using $r_{\mathrm{i}}=r_{\text {coro }}$, which provides an upper bound of the energy dissipation. The circles report the value using $r_{\mathrm{i}}=r_{*}$, which provides the lower bound of the energy dissipation. All the quantities have been computed using temperature and density estimated for the DM Tau system using the results of Guilloteau \& Dutrey (1998), so there is no adjustable parameter in this plot.

using the values listed in Table 1 as input parameters. To remove the problem of our ignorance of the actual value of $r_{\mathrm{i}}$, we have used the relation $r_{*} \leq r_{\mathrm{i}} \leq r_{\text {coro }}$ and computed the corresponding $\dot{M} / \dot{M}_{0}$ and $\mathrm{Re}_{\text {phys }}$. The actual dissipation lies in between the two corresponding estimates. These estimates are plotted in Fig. 5. For comparison, we have added the theoretical predictions (Eqs. (45) and (46)) giving the minimum and the maximum expected values in the turbulent case, as well as the laminar value. This last value is very much lower than the turbulent values, and is never even nearly approached by any stars we considered. This may be seen as a proof of the turbulent character of these disks.

We also see that energy dissipation for disks around T Tauri stars has a tendency to cluster between the minimal and maximal values allowed by the theoretical predictions. The relative position of the cluster of points is slightly better in the case where $r_{\mathrm{i}}$ is computed with $r_{\text {corot }}$, which may be an indication that $r_{\mathrm{i}}$ is actually closer to the corotation radius than to the star radius. However, given the error bars stressed above, this is probably not enough to conclude that disks around T Tauri stars are connected through a magneto-sphere, rather than through a boundary layer. In the case of FU Ori, the points are clearly above the maximum allowed by our choice of parameters. The discrepancy is slightly lower for the case when $r_{\mathrm{i}}=r_{*}$, a choice which should probably be favored by the possible signatures of boundary layer in these objects (Kenyon 1994). In that case, an increase of the surface density by a factor 10 with respect to our values would be enough to solve the discrepancy.

Our estimate neglects the influence of the magnetic field. Laboratory experiments using liquid metals have proved that this can potentially change the intensity of the transport with respect to the pure hydro-dynamical case. However, no experiment has been performed so far to study the magnetic influence in regimes relevant to astrophysical disks.

\subsection{Turbulent viscosity}

The turbulent viscosity in circumstellar disks can be predicted by comparison with laboratory measurements, see Dubrulle et al. (2004). We find:

$v_{t}=\frac{1}{2 \pi} \frac{\tau_{\text {lam }}}{\bar{\tau}} \frac{G}{\operatorname{Re}_{\mathrm{phys}}^{2}}|\bar{S}| \bar{H}^{2}$,

where $\tau=\Sigma S$ is proportional to the angular momentum, and the index lam means laminar value. The ratio $\tau_{\text {lam }} / \tau$ is a function describing the radial variation of the turbulent transport. In an incompressible laboratory flow with constant density, this function is the ratio of the laminar shear profile to the turbulent shear profile: the turbulence regulates itself through a modification of the velocity profile. In Keplerian disks, the shear profile is fixed (through the gravitational force), and the regulation operates through the density. This function has been measured in a number of laboratory experiments. At large Reynolds number, it seems to approach a constant value of 4 predicted by Busse (1970) using the argument of maximal momentum transport. With $|\bar{S}|=1.5 \Omega$ and $G / \operatorname{Re}_{\text {phys }}^{2}=\dot{M} / \dot{M}_{0}$, this defines a resulting typical turbulent viscosity as:

$\bar{v}_{t}=\frac{3}{\pi} \frac{\dot{M}}{\dot{M}_{0}} \bar{\Omega} \bar{H}^{2}$.

This turbulent viscosity takes the shape of an $\alpha$ viscosity, proposed by Shakura \& Sunyaev (1973). The corresponding $\alpha$ coefficient here is a function of the Reynolds number of the circumstellar disk (through Eqs. (45) and (46)). At $\mathrm{Re}_{\mathrm{phys}}=$ $3 \times 10^{13}$, its value is typically $2 \times 10^{-4}<\alpha<2 \times 10^{-2}$. This range is in good agreement with the range of values inferred from the D/H ratio in the Solar System (Drouart et al. 1999; Hersant et al. 2001). More generally, this range is compatible with the disk lifetime and the values usually adopted in theories. Using this expression for the turbulent viscosity, the viscous timescale is:

$t_{v}=\frac{\pi}{3} \frac{\dot{M}_{0}}{\dot{M}}\left(\frac{r}{H}\right)^{2} \frac{1}{\bar{\Omega}}$

\subsection{Energy fluctuations}

Up to now, we have considered only the mean energy dissipation and its luminous counterpart, but we can also obtain interesting information from the luminosity fluctuations which reflect the dynamics of the underlying turbulent flow. In laboratory experiments with smooth boundary conditions, turbulent fluctuations are observed to follow a universal (i.e. Reynolds number-independent), log-normal distribution 


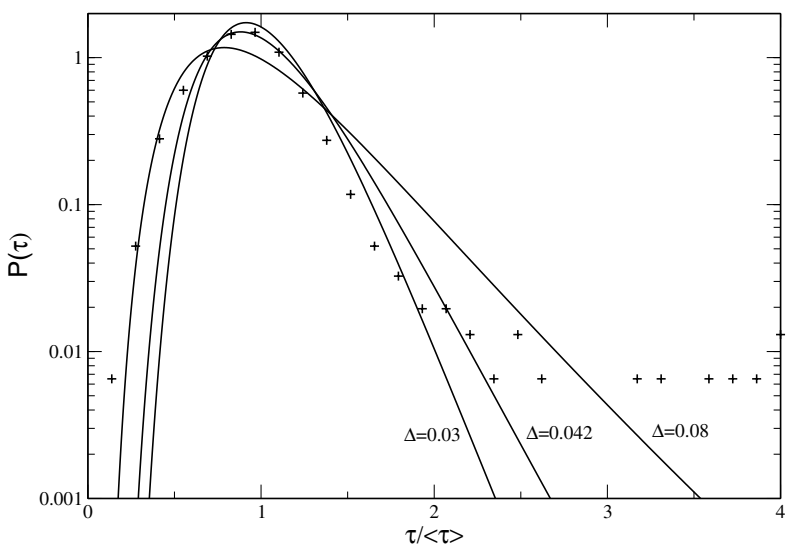

Fig. 6. Distribution of luminosity fluctuations observed disk around BP Tau (symbols) compared with a log-normal distribution of various variance $\Delta$ (plain line). The value of $\Delta$ is indicated beside each line.

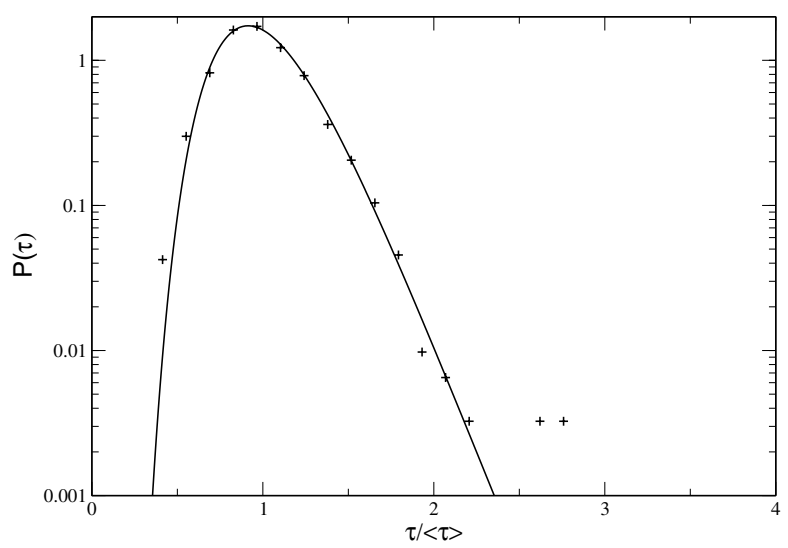

Fig. 7. Distribution of luminosity fluctuations observed disk around V1057 Cyg (symbols) compared with a log-normal distribution of variance $\Delta=0.03$ (plain line).

(Lathrop et al. 1992) with variance $\Delta=0.042$. The universal distribution occurs for variables normalized by their mean. Energy dissipation is proportional to the wall shear stress squared. Since the functional shape of the log-normal distribution is unchanged by squaring, the distribution of energy dissipation should also be log-normal. To check this prediction, we have computed the distribution of the luminosity fluctuations observed from the disk around BP Tau and from the disk around V1057 Cygni. The results are shown in Figs. 6 and 7. One sees that the fluctuations in the disk around V1057 Cyg are very well fitted by a log-normal distribution, with a variance similar to that of laboratory experiments. In the case of BP Tau, however, the comparison is not as good. This difference between the two systems may be traced to different boundary conditions. If we accept that disks around T Tauri stars follow the magnetospheric accretion scenario, while the disk around FU Ori is connected to the star through a boundary layer, it may not be surprising that only the disk around FU Ori follows the laboratory, smooth boundary condition distribution. Since we do not have any measurements for rough boundary conditions, we cannot say whether the discrepancy comes from the different boundary conditions, or from the presence of other physical effects, like accretion shock or a magnetic field.

\subsection{Velocity fluctuations}

Laboratory measurements provide interesting clues about the intensity of velocity fluctuations. Since such fluctuations may be potentially observable in disks using non-thermal line widening, they may be used as additional constraints or observational tests of the analogy between laboratory flows and circumstellar disks. From results of Dubrulle et al. (2004), it appears that azimuthal velocity fluctuations should be proportional to the mean azimuthal velocity, with a proportionality factor depending weakly on the Reynolds number, like $0.03\left(\operatorname{Re} / \operatorname{Re}_{\mathrm{c}}\right)^{-0.125}$. With $\operatorname{Re}_{\text {phys }}=3 \times 10^{13}$ and $\operatorname{Re}_{\mathrm{c}}=10^{8}$ (see Sect. 3.1), the factor is of the order of 0.01. Using Eq. (10), we can compute the azimuthal velocity dispersion for a typical circumstellar disk around a T Tauri. The azimuthal velocity dispersion decreases from about $0.6 \mathrm{~km} \mathrm{~s}^{-1}$ in the inner part, to $0.03 \mathrm{~km} \mathrm{~s}^{-1}$ in the outer part, at $100 \mathrm{AU}$ The total velocity dispersion depends on the anisotropy of the turbulence. In laboratory experiments, the radial relative velocity dispersion is observed to be about twice the azimuthal velocity dispersion. There was no measure of the vertical velocity dispersion, but it can be expected to be much smaller than the horizontal dispersion due to the rotation-induced anisotropy (Dubrulle \& Valdettaro 1992).

The velocity dispersion in disks has been measured by Guilloteau \& Dutrey (1998) at $r>100$ AU They obtain a value of the order of $0.1 \mathrm{~km} \mathrm{~s}^{-1}$, which would correspond to a value of about $0.05 \mathrm{~km} \mathrm{~s}^{-1}$ for the azimuthal component. This is close to the values found from comparison with laboratory flows.

\section{Summary}

In this paper, we have derived and studied the analogy between circumstellar disks and the Taylor-Couette flow. This analogy results in a number of parameter-free predictions about stability of the disks, and their turbulent transport properties, provided an estimate of the disk structure is available. We have proposed to get this estimate from interferometric observations of circumstellar disks, and checked that the energy dissipation, the turbulent transport, and the fluctuations in circumstellar disks all follow behavior compatible with the prediction from the analogy. This check can first be used as a clear proof of the turbulent character of circumstellar disks. A second interesting application would be to build from this analogy a parameterfree model of circumstellar disks. In this respect, the proportionality between the turbulent viscosity and the so-called "accretion rate" (a quantity easily accessible by observation) is very interesting because it opens the possibility to infer the disk structure from the observation of its luminosity. For this, a model has to be built linking the turbulent transport and the disk structure. This is the subject of ongoing work.

We note finally that our model could also possibly apply to other types of disks (e.g. around black holes, or in close binaries) given minor adaptations.

Acknowledgements. We thank A. Dutrey for useful discussions and comments on the manuscript. We are indebted to the anonymous referee whose helpful remarks led us to clarify the paper and our 
thoughts. This work has received support from the Programme national de Planétologie. F.H. acknowledges support from an ESA research fellowship.

\section{References}

D’Alessio, P., Canto, J., Calvet, N., \& Lizano, S. 1998, ApJ, 500, 411 Bahl, S. K. 1970, Def. Sci. J., 20, 89

Barge, P., \& Sommeria, J. 1995, A\&A, 295, L1

van den Berg, T. H., Doering, C. R., Lohse, D., \& Lathrop, D. P. 2003, PRL, submitted

Chavanis, P.-H. 2000, A\&A, 356, 1089

Bouvier, J. 1990, AJ, 99, 946

Bouvier, J., Forestini, M., \& Allain, S. 1997, A\&A, 326, 1023

Busse, F. H. 1970, J. Fluid Mech., 47, 28

Dartois, E., Dutrey, A., \& Guilloteau, S. 2003, A\&A, 399, 773

Dutrey, A., Guilloteau, S., \& Simon, M. 2003, A\&A, 402, 1003

Drouart, A., Dubrulle, B., Gautier, D., \& Robert, F. 1999, Icarus, 140, 129

Dubrulle, B. 1992, A\&A, 266, 592

Dubrulle, B., \& Valdettaro, L. 1992, A\&A, 263, 387

Dubrulle, B., Dauchot, O., Daviaud, F., Longaretti, P.-Y., Richard, D., \& Zahn, J.-P. 2004, Phys. Fluids, submitted

Dubrulle, B., Marié, L., Normand, Ch., Hersant, F., Richard, D., \& Zahn, J.-P. 2005, A\&A, 429, 1

Feigelson, E., \& Montmerle, Th. 1999, ARA\&A, 37, 363

Ferreira, J. 1997, A\&A, 319, 340

Fromang, S., Terquem, C., \& Balbus, S. 2002, MNRAS, 329, 18

Dutrey, A., Guilloteau, S., \& Simon, M. 2003, A\&A, 402, 1003

Guilloteau, S., \& Dutrey, A. 1998, A\&A, 339, 467
Gullbring, E., Hartmann, L., Briceño, C., \& Calvet, N. 1998, ApJ, 492, 323

Hartmann, L., Calvet, N., Gullbring, E., \& D’Alessio, P. 1998, ApJ, 495, 385

Hartmann, L., Calvet, N., Gullbring, E., \& D’Alessio, P. 1998, ApJ, 495,385

Hartmann, L. 2002, ApJ, L29

Hersant, F., Gautier, D., \& Huré, J.-M. 2001, ApJ, 554, 391

Huré, J.-M. 2000, A\&A, 358, 378

Igea, J., \& Glassgold, A. E. 1999, ApJ, 518, 848

Kenyon, S. 1994, ASP Conf. Ser., 56, 63

Kenyon, S. J. 1999, OSPS conf., 613

Lang, K. R., Astrophysical Formulae (Springer-Verlag), 1980

Lathrop, D. P., Fineberg, J., \& Swinney, H. L. 1992, Phys. Rev. A, 46 Longaretti, P.-Y. 2003, preprint

Muzerolle, J., Calvet, N., \& Hartmann, L. 1998, ApJ, 492, 743

Popham, R. 1996, ApJ, 467, 749

Popham, R., Kenyon, S., Hartmann, L., \& Narayan, R. 1996, ApJ, 473, 422

Pringle, J. E. 1981, ARA\&A, 19, 137

Sano, T., Miyama, S. M., Umebayashi, T., \& Nakanon, T. 2000, ApJ, 543,486

Shakura, N. I., \& Sunyaev, R. A. 1973, A\&A, 24, 337

Schatzman, E. 1962, Ann. Astrophys., 25, 18

Schatzman, E. 1989, in Turbulence and non-linear dynamics in MHD flows, ed. M. Meneguzzi, A. Pouquet, \& P. L. Sulem, North-Holland

Tanga, P., Babiano, A., Dubrulle, B., \& Provenzale, A. 1996, Icarus, 121,158

Willis, A. P., \& Barenghi, C. F. 2002, A\&A, 388, 688 